\title{
Solvability of Degenerating Hyperbolic Differential Equations with Unbounded Operator Coefficients
}

\author{
A. V. Glushak ${ }^{1 *}$ \\ ${ }^{1}$ Belgorod State University, Belgorod, 308015 Russia \\ e-mail:*aleglu@mail.ru
}

Received May 29, 2020; revised May 29, 2020; accepted June 26, 2020

\begin{abstract}
We consider initial value problems for a number of hyperbolic equations with a power-law degeneracy and with operator coefficients in a Banach space and establish sufficient conditions for the unique solvability of these problems in terms of the coefficients of the equation, the degeneracy order, and the initial elements.
\end{abstract}

DOI: $10.1134 /$ S0012266121010055

\section{INTRODUCTION}

Differential equations in which the coefficient of the leading derivative has zeros do not fit in the framework of the standard theory of differential equations and have been attracting the interest of a broad research community for a long time (see the monographs [1-3] and the bibliography therein). Although some types of such equations have been comprehensively studied, there are topics (in particular, the construction of closed-form expressions for solutions) requiring further research in the case of second-order equations with operator coefficients (abstract equations) degenerating into first-order equations.

In the present paper, we consider equations in a Banach space in the hyperbolic case. The Cauchy problem for a differential equation of the form $u^{\prime \prime}(t)-t^{\alpha} A u(t)=0, \alpha>0$, where $A=A_{0}^{2}$ and the operator $A_{0}$ is the generator of a $C_{0}$ group, was studied earlier in [4]; the case of a Hilbert space was considered in [5]. The Cauchy problem with the generator of an operator cosine function for a weakly degenerating differential equation of the form $t^{\gamma} u^{\prime \prime}(t)-A u(t)=f(t), 0<\gamma<2$, was considered in [6] (as to the terminology, see, e.g., the monograph [7]). The study of both degenerating equations was carried out by reducing them to an Euler-Poisson-Darboux equation.

We consider a more general form of the differential equation and the operator coefficient (which, in addition, has a variable component) than in [4-6]. The research is also conducted by reduction to a Euler-Poisson-Darboux equation, whereby we need to use the operator Bessel function introduced by the present author [8] and studied in $[8,9]$.

Let us describe the class of operators $A$ to be dealt with below when considering various equations. The papers $[8,9]$ studied the well-posed solvability of the Cauchy problem

$$
\begin{gathered}
v^{\prime \prime}(t)+\frac{k}{t} v^{\prime}(t)=A v(t), \quad t>0, \\
v(0)=u_{0}, \quad v^{\prime}(0)=0
\end{gathered}
$$

with $k>0$ for the Euler-Poisson-Darboux equation with an operator coefficient $A$ in the hyperbolic case.

A necessary and sufficient solvability condition is stated in [8] in terms of estimates for the norm of the resolvent $(\lambda I-A)^{-1}$ and its weighted derivatives, while the paper [9], unlike [8], states a criterion for the uniform well-posedness of this problem in terms of a fractional power of the resolvent and its nonweighted derivatives.

We denote the class of operators $A$ for which the Cauchy problem (1), (2) is uniformly well posed by $G_{k}$ and the corresponding resolving operator (which will be called the Bessel operator function) by $Y_{k}(t)$; i.e., $v(t)=Y_{k}(t) u_{0}$. Further, we denote the set of generators of the cosine operator function $C(t)$ by $G_{0}\left(G_{0} \subset G_{k}\right.$ at $\left.k>0\right)$ and write $Y_{0}(t)=C(t)$. The class $G_{k}$ will 
be referred to as the well-posed solvability class and the number $k$, as its index. Examples of operators $A \in G_{k}$ and the Bessel operator functions $Y_{k}(t)$ generated by them can be found in [9]. In particular, if $A \in G_{0} \subset G_{k}, k>0$, then

$$
Y_{k}(t)=\frac{2 \Gamma(k / 2+1 / 2)}{\Gamma(1 / 2) \Gamma(k / 2)} \int_{0}^{1}\left(1-s^{2}\right)^{k / 2-1} C(t s) d s,
$$

where $\Gamma(\cdot)$ is the Euler gamma function.

\section{WEAKLY DEGENERATING DIFFERENTIAL EQUATION WITH POWER-LAW DEGENERACY}

For $t \geq 0$, consider the weakly degenerating differential equation

$$
t^{\gamma} u^{\prime \prime}(t)+b t^{\gamma-1} u^{\prime}(t)=\left(A+t^{\beta} B\right) u(t)
$$

with operator coefficients in a Banach space $E$, where $0<\gamma<2, b \in \mathbb{R}, \beta \geq 0, A$ is a closed unbounded operator, and $B \in \mathrm{L}(E)$ is a bounded operator. The inclusion $\gamma \in(0,2)$ implies weak degeneracy as opposed to strong degeneracy, where $\gamma \geq 2$, which will also be considered in the present paper.

A solution of Eq. (4) is understood to be a twice continuously differentiable function $u(t)$ on $(0,+\infty)$ that belongs to the domain $D(A)$ of the operator $A$ and satisfies the equation for all $t>0$. We define the solutions of all the equations considered in the present paper in a similar way.

The inhomogeneous equation (4) in the scalar case with $\gamma=1, A=\lambda>0$, and $B=0$ was analyzed by methods of semigroup theory in the paper [10] when studying stochastic processes that are the limits of sequences of random walks.

The change of the independent variable $t=(\tau / \nu)^{\nu}, \nu=2 /(2-\gamma)$ and of the unknown function $u(t)=u\left((\tau / \nu)^{\nu}\right)=w(\tau)$, with allowance for the obvious relations

$$
u^{\prime}(t)=\left(\frac{\tau}{\nu}\right)^{1-\nu} w^{\prime}(\tau), \quad u^{\prime \prime}(t)=\left(\frac{\tau}{\nu}\right)^{2(1-\nu)}\left(w^{\prime \prime}(\tau)+\frac{1-\nu}{\tau} w^{\prime}(\tau)\right),
$$

reduces the weakly degenerating equation (4) to the Euler-Poisson-Darboux equation

$$
w^{\prime \prime}(\tau)+\frac{b \nu-\nu+1}{\tau} w^{\prime}(\tau)=\left(A+\left(\frac{\tau}{\nu}\right)^{\nu \beta} B\right) w(\tau), \quad \tau>0 .
$$

The well-posed statement of initial conditions for the Euler-Poisson-Darboux equation (6) depends on the sign of the parameter $b \nu-\nu+1$. If $b \nu-\nu+1 \geq 0$, then the initial conditions have the form

just as for Eq. (1).

$$
w(0)=u_{0}, \quad w^{\prime}(0)=0
$$

If $b \nu-\nu+1<0$, then one must pose the weighted initial condition (see [11])

$$
w(0)=0, \quad \lim _{\tau \rightarrow 0+} \tau^{b \nu-\nu+1} w^{\prime}(\tau)=u_{1} .
$$

A more general statement of the initial conditions is possible as well (see [12]), but it requires an additional smoothness of the initial elements, and we will not consider it here.

Of course, the solvability of the initial value problem for Eq. (6) also depends on the operator $A+(\tau / \nu)^{\nu \beta} B$. We assume that the operator $A$ belongs to a class $G_{k}$ for some $k>0$, which is broader than in the paper [6] cited in the Introduction $\left(G_{k} \supset G_{0}\right)$.

Let $A \in G_{k}, k>0$. The question as to whether the perturbed operator $A+B$, where $B$ is a bounded operator, belongs to the class $G_{k}$ was studied in [13], and the question as to whether $A+B$, where $B \in G_{m}, m \geq 0$, is an unbounded operator, belongs to some well-posedness class was studied in [14]. These perturbation results considerably broaden the class of operators generating Bessel operator functions. In the present paper, we consider the perturbation of an operator $A \in G_{k}, k>0$, by a variable operator of the form $B(\tau)=(\tau / \nu)^{\nu \beta} B$. 
Theorem 1. Let $k>0$, let $A \in G_{k}$, let $B(t)=(t / \nu)^{\nu \beta} B, \nu \beta \geq 0$, and let $Q(t, s), t \geq s>0$, be a continuous operator satisfying the operator differential equation

$$
\frac{\partial^{2} Q(t, s)}{\partial t^{2}}-\frac{k^{2}-2 k}{4 t^{2}} Q(t, s)-B(t) Q(t, s)=\frac{\partial^{2} Q(t, s)}{\partial s^{2}}-\frac{k^{2}-2 k}{4 s^{2}} Q(t, s)
$$

and the boundary conditions

$$
\frac{d Q(t, t)}{d t}=\frac{1}{2} B(t), \quad \lim _{s \rightarrow 0+} s^{k / 2-1} Q(t, s)=0 .
$$

Then the function

$$
v(t)=Y_{k}(t) u_{0}+t^{-k / 2} \int_{0}^{t} s^{k / 2} Q(t, s) Y_{k}(s) u_{0} d s
$$

is the unique solution of the equation

$$
v^{\prime \prime}(t)+\frac{k}{t} v^{\prime}(t)=(A+B(t)) v(t)
$$

with conditions (2).

Proof. For the first term in the representation (11), by the definition of the Bessel operator function $Y_{k}(t)$, one has the relation

$$
Y_{k}^{\prime \prime}(t) u_{0}+\frac{k}{t} Y_{k}^{\prime}(t) u_{0}=A Y_{k}(t) u_{0}
$$

here and in what follows, we will use the notation

$$
Y_{k}^{\prime}(t) u_{0}=\left(Y_{k}(t) u_{0}\right)^{\prime}
$$

Denote the second term in the representation $(11)$ by $\varphi(t)$; i.e.,

$$
\varphi(t)=t^{-k / 2} \int_{0}^{t} s^{k / 2} Q(t, s) Y_{k}(s) u_{0} d s .
$$

Differentiating identity (14) twice, we obtain

$$
\begin{aligned}
\varphi^{\prime}(t) & =t^{-k / 2} \int_{0}^{t} s^{k / 2}\left(\frac{\partial Q(t, s)}{\partial t}-\frac{k}{2 t} Q(t, s)\right) Y_{k}(s) u_{0} d s+Q(t, t) Y_{k}(t) u_{0}, \\
\varphi^{\prime \prime}(t) & =t^{-k / 2} \int_{0}^{t} s^{k / 2}\left(\frac{\partial^{2} Q(t, s)}{\partial t^{2}}-\frac{k}{t} \frac{\partial Q(t, s)}{\partial t}+\frac{2 k+k^{2}}{4 t^{2}} Q(t, s)\right) Y_{k}(s) u_{0} d s \\
& +\left(\left.\frac{\partial Q(t, s)}{\partial t}\right|_{s=t}+\frac{d Q(t, t)}{d t}-\frac{k}{2 t} Q(t, t)\right) Y_{k}(t) u_{0}+Q(t, t) Y_{k}^{\prime}(t) u_{0},
\end{aligned}
$$

and therefore,

$$
\begin{aligned}
\varphi^{\prime \prime}(t)+\frac{k}{t} \varphi^{\prime}(t)=t^{-k / 2} \int_{0}^{t} s^{k / 2}\left(\frac{\partial^{2} Q(t, s)}{\partial t^{2}}-\frac{k^{2}-2 k}{4 t^{2}} Q(t, s)\right) Y_{k}(s) u_{0} d s & \\
+\left(\left.\frac{\partial Q(t, s)}{\partial t}\right|_{s=t}+\frac{d Q(t, t)}{d t}+\frac{k}{2 t} Q(t, t)\right) Y_{k}(t) u_{0}+Q(t, t) Y_{k}^{\prime}(t) u_{0} . & \text { DIFFERENTIAL EQUATIONS Vol. } 57 \text { No. } 12021
\end{aligned}
$$


We denote the integral in relation (15) by $\psi(t)$ and simplify it by using Eq. (9) and the boundary condition (10). After integrating by parts twice, we obtain

$$
\begin{aligned}
\psi(t)= & \int_{0}^{t} s^{k / 2}\left(\frac{\partial^{2} Q(t, s)}{\partial t^{2}}-\frac{k^{2}-2 k}{4 t^{2}} Q(t, s)\right) Y_{k}(s) u_{0} d s \\
= & \int_{0}^{t} s^{k / 2} \frac{\partial^{2} Q(t, s)}{\partial s^{2}} Y_{k}(s) u_{0} d s+\int_{0}^{t} s^{k / 2}\left(B(t)-\frac{k^{2}-2 k}{4 s^{2}} I\right) Q(t, s) Y_{k}(s) u_{0} d s \\
= & \left.t^{k / 2} \frac{\partial Q(t, s)}{\partial s}\right|_{s=t} Y_{k}(t) u_{0}-\int_{0}^{t} \frac{\partial Q(t, s)}{\partial s}\left(\frac{k}{2} s^{k / 2-1} Y_{k}(s)+s^{k / 2} Y_{k}^{\prime}(s)\right) u_{0} d s \\
& \quad+\int_{0}^{t} s^{k / 2}\left(B(t)-\frac{k^{2}-2 k}{4 s^{2}} I\right) Q(t, s) Y_{k}(s) u_{0} d s \\
= & \left.t^{k / 2} \frac{\partial Q(t, s)}{\partial s}\right|_{s=t} Y_{k}(t) u_{0}-\frac{k t^{k / 2-1}}{2} Q(t, t) Y_{k}(t) u_{0}-t^{k / 2} Q(t, t) Y_{k}^{\prime}(t) u_{0} \\
& +\int_{0}^{t} s^{k / 2} Q(t, s)\left(Y_{k}^{\prime \prime}(s)+\frac{k}{s} Y_{k}^{\prime}(s)\right) u_{0} d s+\int_{0}^{t} s^{k / 2} B(t) Q(t, s) Y_{k}(s) u_{0} d s .
\end{aligned}
$$

Replacing the integral in (15) with its representation (16), by virtue of the boundary condition (10) we arrive at the relation

$$
\begin{aligned}
\varphi^{\prime \prime}(t)+\frac{k}{t} \varphi^{\prime}(t)= & t^{-k / 2}(A+B(t)) \int_{0}^{t} s^{k / 2} Q(t, s) Y_{k}(s) u_{0} d s \\
& +\left(\left.\frac{\partial Q(t, s)}{\partial s}\right|_{s=t}+\left.\frac{\partial Q(t, s)}{\partial t}\right|_{s=t}+\frac{d Q(t, t)}{d t}\right) Y_{k}(t) u_{0} \\
= & (A+B(t)) \varphi(t)+2 \frac{d Q(t, t)}{d t} Y_{k}(t) u_{0}=(A+B(t)) \varphi(t)+B(t) Y_{k}(t) u_{0} .
\end{aligned}
$$

In view of relations (13)-(17), we eventually obtain

$$
v^{\prime \prime}(t)+\frac{k}{t} v^{\prime}(t)=A Y_{k}(t) u_{0}+(A+B(t)) \varphi(t)+B(t) Y_{k}(t) u_{0}=(A+B(t)) v(t) .
$$

Thus, the function $v(t)$ defined in (11), which, for convenience, will be denoted by

$$
v(t)=\tilde{Y}_{k}(t) u_{0} \equiv Y_{k}(t) u_{0}+t^{-k / 2} \int_{0}^{t} s^{k / 2} Q(t, s) Y_{k}(s) u_{0} d s
$$

in what follows, is a solution of Eq. (12).

One can readily verify that the function $v(t)$ satisfies conditions (2). To this end, write the function $v(t)$ in the form

$$
v(t)=Y_{k}(t) u_{0}+t \int_{0}^{1} \xi^{k / 2} Q(t, t \xi) Y_{k}(t \xi) u_{0} d \xi
$$


and take into account the properties $Y_{k}(0)=I$ and $Y_{k}^{\prime}(0)=0$ of the Bessel operator function as well as the relation

$$
\lim _{t \rightarrow 0+} Q(t, t \xi)=0
$$

which follows from Eq. (9).

The uniqueness of the solution of problem (9), (10) is proved by contradiction. Let $v_{1}(t)$ and $v_{2}(t)$ be two solutions of problem (9), (10). Consider the function

$$
V(t, s)=f\left(\tilde{Y}_{k}(s)\left(v_{1}(t)-v_{2}(t)\right)\right)
$$

of two variables, where $f \in E^{*}\left(E^{*}\right.$ is the dual space of $E$ ) and $t, s \geq 0$. Obviously, this function satisfies the equation

$$
\frac{\partial^{2} V}{\partial t^{2}}+\frac{k}{t} \frac{\partial V}{\partial t}=\frac{\partial^{2} V}{\partial s^{2}}+\frac{k}{s} \frac{\partial V}{\partial s}, \quad t, s>0
$$

and the initial conditions

$$
V(0, s)=\frac{\partial V(0, s)}{\partial t}=0
$$

This problem for a partial differential equation is reduced by the change of variables (see [15, Sec. 5, item 3])

$$
t_{1}=(t+s)^{2} / 4, \quad s_{1}=(t-s)^{2} / 4
$$

to a problem for which the uniqueness of a solution in the class of functions twice continuously differentiable for $t, s \geq 0$ has been established in [15, Sec. 5, item 2]). Moreover, the statement on the uniqueness is also contained in Theorem 6.1 in the paper [16], which even treats a more general equation.

The explicit formula obtained in the paper [15] for the solution of the above problem implies the identity $V(t, s) \equiv 0$. Since $f \in E^{*}$ is arbitrary, by setting $s=0$ we obtain $v_{1}(t) \equiv v_{2}(t)$, and the uniqueness of solution is thus established. The proof of the theorem is complete.

As was proved in Theorem 1, the solvability of the initial value problem for Eq. (12) depends on the solvability of the boundary value problem (9), (10) for an operator differential equation, which has essentially been established in the paper [17].

Theorem 2. The operator differential equation (9) with the boundary conditions (10) has a solution that can be obtained by the successive approximation method.

For $B \in \mathbb{R}$, the solvability of the boundary value problem for the scalar equation (9) was established in [17]. One can readily verify that replacing the coefficient $B \in \mathbb{R}$ with a continuous operator coefficient $B(t)=(t / \nu)^{\nu \beta} B$ does not invalidate the reasoning in Secs. 2, 3 in the paper [17]. Note also that the case of $k=0$ was considered in the paper [18].

Let us also point out that if $\beta=0, k=2 n$, and $n \in \mathbb{N}$, then, by Theorem 3 in [13], the function $Q(t, s)$ can be written in closed form as

$$
Q(t, s)=\frac{s^{n} B}{2^{n+1} n ! t^{n-1}}\left(\frac{1}{s} \frac{d}{d s}\right)^{n}\left(\left(s^{2}-t^{2}\right)_{1}^{n} F_{2}\left(1 ; n+1,2 ; \frac{t^{2}-s^{2}}{4} B\right)\right),
$$

where ${ }_{1} F_{2}(\cdot)$ is the generalized hypergeometric function.

Having established the results on the solvability of the initial value problem for the perturbed Euler-Poisson-Darboux equation (12), let us now state results on the solvability of initial value problems for the weakly degenerating equation (4).

Let $b \nu-\nu+1 \geq 0$ and $\nu=2 /(2-\gamma)$, or, equivalently, $2 b \geq \gamma$. Returning to the original variable $t$ and the unknown function $u(t)$, we conclude that Eq. (6) is transformed into Eq. (4) and the initial conditions (7), by virtue of relations (5), are transformed into the conditions

$$
u(0)=u_{0}, \quad \lim _{t \rightarrow 0+} t^{1-1 / \nu} u^{\prime}(t)=0 .
$$


Theorems 1 and 2 imply the following assertion on the solvability of the initial value problem (4), (19).

Theorem 3. Let $0<\gamma<2,2 b \geq \gamma, \beta \geq 0, A \in G_{(2 b-\gamma) /(2-\gamma)}, B \in \mathrm{L}(E)$, and $u_{0} \in D(A)$. Then the function $u(t)=\tilde{Y}_{(2 b-\gamma) /(2-\gamma)}\left(\nu t^{1 / \nu}\right) u_{0}$, where $\nu=2 /(2-\gamma)$ and the function $\tilde{Y}_{(2 b-\gamma) /(2-\gamma)}(\cdot)$ is defined by relation (18), is the unique solution of Eq. (4) with the initial conditions (19).

Now let $b \nu-\nu+1<0$ or $2 b<\gamma$. In this case, for Eq. (6) one should pose the initial conditions (8), which for the original function $u(t)$ become

$$
u(0)=0, \quad \nu^{b \nu-\nu+1} \lim _{t \rightarrow 0+} t^{b} u^{\prime}(t)=u_{1}
$$

Considering the results in [11] on the solvability of the weighted Cauchy problem for the EulerPoisson-Darboux equation, we arrive at the following assertion.

Theorem 4. Let $0<\gamma<2,2 b<\gamma, \beta \geq 0, A \in G_{(4-b-\gamma) /(2-\gamma)}, B \in \mathrm{L}(E)$, and $u_{1} \in D(A)$. Then the function

$$
u(t)=\frac{\nu^{\nu-b \nu-1} t^{1-b}}{1-b} \tilde{Y}_{(4-b-\gamma) /(2-\gamma)}\left(\nu t^{1 / \nu}\right) u_{1}
$$

where $\nu=2 /(2-\gamma)$ and the function $\tilde{Y}_{(4-b-\gamma) /(2-\gamma)}(\cdot)$ is defined by relation $(18)$, is the unique solution of Eq. (4) with the initial conditions (20).

In particular, for $b=0$ and $B=0$ with an operator $A \in G_{\nu+1}$ in the broader set $G_{\nu+1} \supset G_{0}$ than in [6], the solution of problem (4), (20) has the form

$$
u(t)=\nu^{\nu-1} t Y_{\nu+1}\left(\nu t^{1 / \nu}\right) u_{1} .
$$

The assertions in Theorems 3 and 4 also obviously hold for $\gamma=0$, because Eq. (4) is already an Euler-Poisson-Darboux equation in this case. These theorems not only specify the statement of initial conditions and prove the unique solvability of the corresponding initial problems for Eq. (4) but also establish a connection between the degeneracy order $\gamma$, the coefficient $b$ multiplying the first derivative $u^{\prime}(t)$, and the set of operators $A$ forming the well-posed solvability class.

An elementary analysis of the assertions in Theorem 3 leads to the following conclusions. If $0 \leq \gamma \leq 2 b$ and $0 \leq b<1$, then the index of the well-posed solvability class with a fixed $b$ decreases in the variable $\gamma$ from $b$ to 0 , with the well-posed solvability class itself narrowed down from $G_{b}$ to $G_{0}$. If $0 \leq \gamma<2$ and $b=1$, then the well-posed solvability class is one and the same for all $\gamma$ and coincides with $G_{1}$. If $0 \leq \gamma<2$ and $b>1$, then the index of the well-posed solvability class increases in the variable $\gamma$ from $\bar{b}$ to $+\infty$. Under the condition $2 b=\gamma, 0 \leq \gamma<2$, the well-posed solvability class is one and the same for all $\gamma$ and coincides with $G_{0}$.

For a fixed $\gamma, 0 \leq \gamma<2$, the index of the well-posed solvability class increases in the variable $b$ from $\gamma / 2$ to $+\infty$.

Under the conditions $2 b=\gamma, 0 \leq \gamma<2$, and $B=0$, the well-posed solvability class is one and the same for all $\gamma$ and coincides with $G_{0}$. The limit case of $b=\gamma=0$ corresponds to the abstract wave equation

$$
u^{\prime \prime}(t)=A u(t), \quad t \geq 0, \quad A \in G_{0},
$$

which is not degenerating. It is well known that the unique solution of this equation with the initial conditions

$$
u(0)=u_{0}, \quad u^{\prime}(0)=u_{1}, \quad u_{0}, u_{1} \in D(A),
$$

is the function $u(t)=C(t) u_{0}+S(t) u_{1}$, where $C(t)$ is the cosine operator function and

$$
S(t)=\int_{0}^{t} C(\tau) d \tau
$$

is the sine operator function. 
Another limit case of $\gamma=2, b=1$, which does not reduce to an Euler-Poisson-Darboux equation under the change of variables in Sec. 1 and which has not been studied in Sec. 1, results in the degenerating abstract Euler equation

$$
t^{2} u^{\prime \prime}(t)+t u^{\prime}(t)=A u(t), \quad t>0,
$$

which, for $A \in G_{0}$, has the unique solution

$$
u(t)=C(\ln t) u_{0}+S(\ln t) u_{1}
$$

with the initial conditions

$$
u(1)=u_{0}, \quad u^{\prime}(1)=u_{1}, \quad u_{0}, u_{1} \in D(A) .
$$

In the monograph [19], a solution bounded at the point of degeneracy of the inhomogeneous abstract Euler equation with operator coefficients is found by the method of small stabilizing perturbations.

A similar analysis can be carried out using Theorem 4 .

\section{ABSTRACT SHARP EQUATION}

Now consider the special case of Eq. (4) for $b=\gamma=\beta=1$ and $B=-I$. The equation

$$
t u^{\prime \prime}(t)+u^{\prime}(t)-t u(t)=A_{0} u(t)
$$

is called Sharp's equation (see [20, p. 118]), and Theorem 3 holds for this equation with $A_{0} \in G_{1}$. Let us show that an explicit formula can also be specified for some its solution in the case where $A_{0} \notin G_{1}$. Naturally, problem (4), (19) with such an operator will not be well posed, because the inclusion $A_{0} \in G_{1}$ is a necessary and sufficient condition for well-posedness.

Let the operator $A_{0}$ generate a uniformly bounded group $T\left(t ; A_{0}\right)$; then $A_{0}^{2} \in G_{0}$ and $C\left(t ; A_{0}^{2}\right)=1 / 2\left(T\left(t ; A_{0}\right)+T\left(-t ; A_{0}\right)\right)$ is the uniformly bounded cosine operator function generated by this operator.

For $u_{2} \in D\left(A_{0}^{2}\right)$, we introduce the function

$$
\begin{aligned}
u(t)=\int_{0}^{\pi / 2} \cosh (t & \cos \varphi) C\left(\ln \cot \frac{\varphi}{2} ; A_{0}^{2}\right) u_{2} d \varphi \\
& +\int_{0}^{\pi / 2} \sinh (t \cos \varphi) A_{0} S\left(\ln \cot \frac{\varphi}{2} ; A_{0}^{2}\right) u_{2} d \varphi
\end{aligned}
$$

where $S\left(t ; A_{0}^{2}\right)$ is the sine operator function.

By virtue of the uniform boundedness of the cosine operator function $C\left(t ; A_{0}^{2}\right)$, the convergence of the first integral in (22) is obvious, while the convergence of the second integral follows from the finiteness of integral (2.6.34.3 in [21])

$$
\int_{0}^{\pi / 2} \ln \sin \frac{\varphi}{2} d \varphi .
$$

Let us show that the function $u(t)$ defined by relation (22) is a solution of Eq. (21) bounded at zero, and, with this aim in mind, calculate its derivatives. After integration by parts, with allowance 
for the uniform boundedness of the cosine operator function, we obtain

$$
\begin{aligned}
& u^{\prime}(t)=\int_{0}^{\pi / 2} \sinh (t \cos \varphi) \cos \varphi C\left(\ln \cot \frac{\varphi}{2} ; A_{0}^{2}\right) u_{2} d \varphi+\int_{0}^{\pi / 2} \cosh (t \cos \varphi) \cos \varphi A_{0} S\left(\ln \cot \frac{\varphi}{2} ; A_{0}^{2}\right) u_{2} d \varphi \\
& =t \int_{0}^{\pi / 2} \cosh (t \cos \varphi) \sin ^{2} \varphi C\left(\ln \cot \frac{\varphi}{2} ; A_{0}^{2}\right) u_{2} d \varphi+\int_{0}^{\pi / 2} \sinh (t \cos \varphi) C^{\prime}\left(\ln \cot \frac{\varphi}{2} ; A_{0}^{2}\right) u_{2} d \varphi \\
& +t \int_{0}^{\pi / 2} \sinh (t \cos \varphi) \sin ^{2} \varphi A_{0} S\left(\ln \cot \frac{\varphi}{2} ; A_{0}^{2}\right) u_{2} d \varphi \\
& +\int_{0}^{\pi / 2} \cosh (t \cos \varphi) A_{0} C\left(\ln \cot \frac{\varphi}{2} ; A_{0}^{2}\right) u_{2} d \varphi \\
& u^{\prime \prime}(t)=\int_{0}^{\pi / 2} \cosh (t \cos \varphi) \cos ^{2} \varphi C\left(\ln \cot \frac{\varphi}{2} ; A_{0}^{2}\right) u_{2} d \varphi \\
& +\int_{0}^{\pi / 2} \sinh (t \cos \varphi) \cos ^{2} \varphi A_{0} S\left(\ln \cot \frac{\varphi}{2} ; A_{0}^{2}\right) u_{2} d \varphi \\
& t u^{\prime \prime}(t)+u^{\prime}(t)=t u(t)+\int_{0}^{\pi / 2} \sinh (t \cos \varphi) A_{0}^{2} S\left(\ln \cot \frac{\varphi}{2} ; A_{0}^{2}\right) u_{2} d \varphi \\
& +\int_{0}^{\pi / 2} \cosh (t \cos \varphi) A_{0} C\left(\ln \cot \frac{\varphi}{2} ; A_{0}^{2}\right) u_{2} d \varphi=t u(t)+A_{0} u(t) .
\end{aligned}
$$

Thus, the bounded function $u(t)$ defined by relation (22) satisfies Eq. (21) and, for $t=0$, the condition

$$
u(0)=\int_{0}^{\pi / 2} C\left(\ln \cot \frac{\varphi}{2} ; A_{0}^{2}\right) u_{2} d \varphi
$$

If we pose the problem of finding a bounded solution of Eq. (21) with the initial condition

$$
u(0)=u_{0},
$$

then for the element $u_{2} \in D\left(A_{0}^{2}\right)$ we obtain the operator equation of the first kind

$$
\int_{0}^{\pi / 2} C\left(\ln \cot \frac{\varphi}{2} ; A_{0}^{2}\right) u_{2} d \varphi=u_{0}
$$

To solve this equation, it is necessary to impose additional smoothness conditions on the initial element $u_{0}$.

Taking into account the representation of the cosine operator function $C\left(t ; A_{0}^{2}\right)$ via the resolvent,

$$
C\left(t ; A_{0}^{2}\right) u_{2}=\frac{1}{2 \pi i} \int_{\sigma-i \infty}^{\sigma+i \infty} e^{\lambda t} \lambda R\left(\lambda^{2} ; A_{0}^{2}\right) u_{2} d \lambda, \quad \sigma>0, \quad u_{2} \in E,
$$


we write the left-hand side of the operator equation (24) in the form

$$
\begin{aligned}
\int_{0}^{\pi / 2} C\left(\ln \cot \frac{\varphi}{2} ; A_{0}^{2}\right) u_{2} d \varphi & =2 \int_{0}^{\infty} \frac{e^{t}}{1+e^{2 t}} C\left(t ; A_{0}^{2}\right) u_{2} d \varphi \\
& =\frac{1}{\pi i} \int_{\sigma-i \infty}^{\sigma+i \infty} \int_{0}^{\infty} \frac{e^{t(\lambda+1)} d t}{1+e^{2 t}} \lambda R\left(\lambda^{2} ; A_{0}^{2}\right) u_{2} d \lambda \\
& =\frac{1}{2 \pi i} \int_{\sigma-i \infty}^{\sigma+i \infty} \beta\left(\frac{1-\lambda}{2}\right) \lambda R\left(\lambda^{2} ; A_{0}^{2}\right) u_{2} d \lambda
\end{aligned}
$$

(Here we have used integral 2.3.12.6 in [21], where

$$
\beta(z)=\frac{1}{2}\left(\psi\left(\frac{z+1}{2}\right)-\psi\left(\frac{z}{2}\right)\right)
$$

and $\psi(\cdot)$ is the psi-function (see, e.g., [21, p. $775 ; 22$, p. 536].)

Using the representation (25), we write the operator equation (24) for $u_{2} \in D\left(A_{0}^{2}\right)$ in the form

$$
P u_{2} \equiv \frac{1}{2 \pi i} \int_{\sigma-i \infty}^{\sigma+i \infty} \beta\left(\frac{1-\lambda}{2}\right) \lambda R\left(\lambda^{2} ; A_{0}^{2}\right) u_{2} d \lambda=u_{0} .
$$

Thus, the question of the solvability of the operator equation (24) reduces to the question of the existence of an inverse operator defined on some subset in $D\left(A_{0}^{2}\right)$ for the bounded operator $P: D\left(A_{0}^{2}\right) \rightarrow E$ given by the left-hand side of Eq. (26) and extended by continuity to $E$. Here an important role is played by the function

$$
\chi(\lambda)=\frac{1}{2} \beta\left(\frac{1-\sqrt{\lambda}}{2}\right),
$$

using which, we write Eq. (26) in the form

$$
P u_{2} \equiv \frac{1}{\pi i} \int_{\sigma-i \infty}^{\sigma+i \infty} \xi \chi\left(\xi^{2}\right) R\left(\xi^{2} ; A_{0}^{2}\right) u_{2} d \xi=u_{0} .
$$

The operator $A_{0}$ generates a uniformly bounded group $T\left(t ; A_{0}\right)$; consequently, the spectrum of the operator $A_{0}^{2}$ lies on the negative half-axis, and therefore, as will be seen from the proof to follow, the fact of the lack [22, p. 536] of real zeros of the function $\chi(\lambda)$ will be of importance for us.

Let $\Upsilon_{1}$ be a contour on the complex plane consisting of the straight line $\operatorname{Re} z=\sigma_{1}>0$ traversed from bottom to top; then $\Upsilon_{1}^{2}$ is a parabola that is the image of the straight line $\Upsilon_{1}$ under the mapping $w=z^{2}\left(z \in \Upsilon_{1}, w \in \Upsilon_{1}^{2}\right)$. Since the spectrum of the operator $A_{0}^{2}$ lies on the negative half-axis, we introduce a contour $\Xi$ obtained from $\Upsilon_{1}$ by subtending towards the negative half-axis so that it does not contain the zeros of the function $\chi(z)$ to the left of itself.

We take a $\lambda_{0}$ such that $\operatorname{Re} \lambda_{0}>\sigma_{1}>0$ from the regular set $\rho\left(A_{0}^{2}\right)$ and introduce the bounded operator

$$
H v=\frac{1}{2 \pi i} \int_{\Xi} \frac{R\left(z ; A_{0}^{2}\right) v d z}{\chi(z)\left(z-\lambda_{0}\right)}, \quad H: E \rightarrow E,
$$

whose absolute convergence follows from the well-known inequality

$$
\left\|\lambda R\left(\lambda^{2} ; A_{0}^{2}\right)\right\| \leq \frac{M}{\operatorname{Re} \lambda-\omega}, \quad \operatorname{Re} \lambda>\omega
$$

for the resolvent of the generator of the cosine operator function $C\left(t ; A_{0}^{2}\right)$. 
Now let us show that the operator $P$ has an inverse operator $P^{-1}: D\left(A_{0}^{2}\right) \rightarrow E$. Let $v \in D\left(A_{0}^{2}\right)$, $\sigma_{1}<\sigma_{2}<\operatorname{Re} \lambda$. Then, applying the operator $H$ defined by relation (27) to $P v$ and considering the Hilbert identity

$$
R\left(z ; A_{0}^{2}\right) R\left(\xi^{2} ; A_{0}^{2}\right)=\frac{R\left(z ; A_{0}^{2}\right)-R\left(\xi^{2} ; A_{0}^{2}\right)}{\xi^{2}-z},
$$

we obtain the relation

$$
\begin{aligned}
H P v & =\frac{1}{2 \pi i} \int_{\Xi} \frac{R\left(z ; A_{0}^{2}\right)}{\chi(z)\left(z-\lambda_{0}\right)} \frac{1}{\pi i} \int_{\sigma_{2}-i \infty}^{\sigma_{2}+i \infty} \xi \chi\left(\xi^{2}\right) R\left(\xi^{2} ; A_{0}^{2}\right) v d \xi \\
& =\frac{2}{(2 \pi i)^{2}} \int_{\Xi} \int_{\sigma_{2}-i \infty}^{\sigma_{2}+i \infty}\left(\frac{\xi \chi\left(\xi^{2}\right) R\left(z ; A_{0}^{2}\right) v}{\chi(z)\left(z-\lambda_{0}\right)\left(\xi^{2}-z\right)}-\frac{\xi \chi\left(\xi^{2}\right) R\left(\xi^{2} ; A_{0}^{2}\right) v}{\chi(z)\left(z-\lambda_{0}\right)\left(\xi^{2}-z\right)}\right) d \xi d z .
\end{aligned}
$$

The integral in (28) converges absolutely. Changing the order of integration, we obtain

$$
\begin{aligned}
H P v= & \frac{2}{(2 \pi i)^{2}} \int_{\Xi} \int_{\sigma_{2}-i \infty}^{\sigma_{2}+i \infty} \frac{\xi \chi\left(\xi^{2}\right) R\left(z ; A_{0}^{2}\right) v d \xi d z}{\chi(z)\left(z-\lambda_{0}\right)\left(\xi^{2}-z\right)} \\
& -\frac{2}{(2 \pi i)^{2}} \int_{\sigma_{2}-i \infty}^{\sigma_{2}+i \infty} \xi \chi\left(\xi^{2}\right) R\left(\xi^{2} ; A_{0}^{2}\right) v \int_{\Xi} \frac{d z}{\chi(z)\left(z-\lambda_{0}\right)\left(\xi^{2}-z\right)} d \xi .
\end{aligned}
$$

If the integration contour $\Xi$ is closed to the left, then the inner integral in the second term in (29) vanishes by the Cauchy theorem. At the same time, we use the Cauchy integral formula to calculate the integrals in the first term in (29). Thus, the relation

$$
\begin{aligned}
H P v & =\frac{2}{(2 \pi i)^{2}} \int_{\Xi} \int_{\Upsilon_{2}} \frac{\xi \chi\left(\xi^{2}\right) R\left(z ; A_{0}^{2}\right) v d \xi d z}{\chi(z)\left(z-\lambda_{0}\right)\left(\xi^{2}-z\right)}=\frac{1}{(2 \pi i)^{2}} \int_{\Xi} \int_{\Upsilon_{2}^{2}} \frac{\chi_{k}(\lambda) R\left(z ; A_{0}^{2}\right) v d \lambda d z}{\chi(z)\left(z-\lambda_{0}\right)(\lambda-z)} \\
& =\frac{1}{2 \pi i} \int_{\Xi} \frac{R\left(z ; A_{0}^{2}\right) v d z}{z-\lambda_{0}}=-R\left(\lambda_{0} ; A_{0}^{2}\right) v
\end{aligned}
$$

holds, where $\Upsilon_{2}$ is the contour on the complex plane consisting of the straight line $\operatorname{Re} z=\sigma_{2}$, $0<\sigma_{1}<\sigma_{2}<\operatorname{Re} \lambda$ traversed from bottom to top, while the contour $\Upsilon_{2}^{2}$ is the parabola that is the image of the contour $\Upsilon_{2}$ under the mapping $w=z^{2}\left(z \in \Upsilon_{2}, w \in \Upsilon_{2}^{2}\right)$.

The pairwise commuting operators $H, P$, and $R\left(\lambda_{0} ; A_{0}^{2}\right)$ are bounded, and the domain $D\left(A_{0}^{2}\right)$ is dense in $E$; therefore, the relation $H P v=-R\left(\lambda_{0} ; A_{0}^{2}\right) v$ also holds for $v \in E$ with $H P: E \rightarrow D\left(A_{0}^{2}\right)$. It follows that the operator $P^{-1} v=-\left(\lambda_{0} I-A_{0}^{2}\right) H v$ for $v \in D\left(A_{0}^{2}\right)$ is the inverse of the operator $P, P^{-1}: D\left(A_{0}^{2}\right) \rightarrow E$. Indeed,

$$
\begin{aligned}
& P P^{-1} v=-P\left(\lambda_{0} I-A_{0}^{2}\right) H v=-P H\left(\lambda_{0} I-A_{0}^{2}\right) v=R\left(\lambda_{0} ; A_{0}^{2}\right)\left(\lambda_{0} I-A_{0}^{2}\right) v=v, \quad v \in D\left(A_{0}^{2}\right), \\
& P^{-1} P v=-\left(\lambda_{0} I-A_{0}^{2}\right) H P v=\left(\lambda_{0} I-A_{0}^{2}\right) R\left(\lambda_{0} ; A_{0}^{2}\right) v=v, \quad v \in E .
\end{aligned}
$$

Returning to the operator equation (26) and additionally demanding that the inclusion $u_{0} \in D\left(A_{0}^{4}\right)$ hold, we determine the initial element

$$
u_{2}=\left(A_{0}^{2}-\lambda_{0} I\right) H u_{0}
$$

belonging to $D\left(A_{0}^{2}\right)$, where the operator $H$ is given by relation (27), $\lambda_{0} \in \rho\left(A_{0}^{2}\right)$, and $\operatorname{Re} \lambda_{0}>\sigma_{1}>0$. Then the function $u(t)$ defined by (22) will be a bounded solution of Eq. (21) with the initial condition (23). Note that the degenerating equation (21) can have a second solution unbounded at zero. Thus, we have established the following assertion. 
Theorem 5. Let an operator $A_{0}$ generate a uniformly bounded group $T\left(t ; A_{0}\right)$, and let the inclusion $u_{0} \in D\left(A_{0}^{4}\right)$ hold. Then the function $u(t)$ defined by relation $(22)$ in which $u_{2}=\left(A_{0}^{2}-\lambda_{0} I\right) H u_{0}$, where the operator $H$ is given by relation (27), $\lambda_{0} \in \rho\left(A_{0}^{2}\right)$, and $\operatorname{Re} \lambda_{0}>\sigma_{1}>0$, is a bounded solution of Eq. (21) with the initial condition (23).

Example 1. Let $E=\mathbb{C}=D(A), A=i A_{1}, A_{1} \in \mathbb{R}$, and $u_{0} \in \mathbb{C}$. Then $T\left(t ; A_{0}\right)=e^{i t A_{1}}$, $C\left(t ; A_{0}^{2}\right)=\cos \left(t A_{1}\right)$, and the solution of problem (21), (23) has the form

$$
u(t)=u_{2} \int_{0}^{\pi / 2} \cosh \left(t \cos \varphi+i A_{1} \ln \cot \frac{\varphi}{2}\right) d \varphi,
$$

where the function $u_{2}$ is found from the condition

$$
u_{2} \int_{0}^{\pi / 2} \cos \left(A_{1} \ln \cot \frac{\varphi}{2}\right) d \varphi=u_{0} .
$$

Example 2. Let $E=B U C(\mathbb{R})$ be the space of bounded uniformly continuous functions on $\mathbb{R}$ (or $\left.E=L^{p}(\mathbb{R}), 1 \leq p<\infty\right)$, and consider the operator $A_{0} u(x)=u^{\prime}(x)$ with the domain $D\left(A_{0}\right)=$ $\left\{u(x) \in E: u(x)\right.$ is absolutely continuous, $\left.u^{\prime}(x) \in E\right\}$. Then

$$
T\left(t ; A_{0}\right) u(x)=u(x+t), \quad C\left(t ; A_{0}^{2}\right) u(x)=1 / 2(u(x+t)+u(x-t)),
$$

and if $u_{2}(x) \in D\left(A_{0}^{2}\right)$, then the function

$$
\begin{aligned}
u(t)=\frac{1}{2} \int_{0}^{\pi / 2}(\cosh (t \cos \varphi)+\sinh (t \cos \varphi)) u_{2}\left(x+\ln \cot \frac{\varphi}{2}\right) d \varphi \\
\quad+\frac{1}{2} \int_{0}^{\pi / 2}(\cosh (t \cos \varphi)-\sinh (t \cos \varphi)) u_{2}\left(x-\ln \cot \frac{\varphi}{2}\right) d \varphi
\end{aligned}
$$

is a bounded solution of Eq. (21).

\section{STRONGLY DEGENERATING DIFFERENTIAL EQUATION WITH POWER-LAW DEGENERACY}

Consider Eq. (4) in the case of strong degeneracy, $\gamma>2$. The change of the independent variable $t=(-\tau / \nu)^{-\nu}, \nu=2 /(2-\gamma)$, and of the unknown function $u(t)=u\left((-\tau / \nu)^{-\nu}\right)=w(\tau)$ transforms the strongly degenerating equation (4) into the Euler-Poisson-Darboux equation

$$
w^{\prime \prime}(\tau)+\frac{1+\nu-b \nu}{\tau} w^{\prime}(\tau)=\left(A+\left(-\frac{\tau}{\nu}\right)^{-\nu \beta} B\right) w(\tau), \quad \tau>0 .
$$

By analogy with Theorems 3 and 4, one can establish the following Theorems 6 and 7, in which the statement of initial conditions in the case of strong degeneracy is specified, the unique solvability of the corresponding initial value problems for Eq. (4) is proved, and the relation between the degeneracy order $\gamma$, the coefficient $b$ multiplying the first derivative $u^{\prime}(t)$, and the set of operators $A$ forming the well-posed solvability class is established.

Theorem 6. Let $\gamma>2,2 b \geq 4-\gamma, \beta \geq 0, A \in G_{(2 b+\gamma-4) /(\gamma-2)}, B \in \mathrm{L}(E)$, and $u_{0} \in D(A)$. Then the function $u(t)=\tilde{Y}_{(2 b+\gamma-4) /(\gamma-2)}\left(-\nu t^{-1 / \nu}\right) u_{0}$, where $\nu=2 /(2-\gamma)$ and the function $\tilde{Y}_{(2 b+\gamma-4) /(\gamma-2)}(\cdot)$ is defined by relation (18), is the unique solution of Eq. (4) with the initial conditions

$$
u(0)=u_{0}, \quad \lim _{t \rightarrow 0+} t^{1+1 / \nu} u^{\prime}(t)=0 .
$$


Theorem 7. Let $\gamma>2,2 b<4-\gamma, \beta \geq 0, A \in G_{(\gamma-2 b) /(\gamma-2)}, B \in \mathrm{L}(E)$, and $u_{1} \in D(A)$. Then the function

$$
u(t)=\frac{(-\nu)^{b \nu-\nu-1} t^{1-b}}{1-b} \tilde{Y}_{(\gamma-2 b) /(\gamma-2)}\left(-\nu t^{-1 / \nu}\right) u_{1}
$$

where $\nu=2 /(2-\gamma)$ and the function $\tilde{Y}_{(\gamma-2 b) /(\gamma-2)}(\cdot)$ is defined by relation (18), is the unique solution of Eq. (4) with the initial conditions

$$
u(0)=0, \quad(-\nu)^{1+\nu-b \nu} \lim _{t \rightarrow 0+} t^{b} u^{\prime}(t)=u_{1} .
$$

\section{ABSTRACT ANALOG OF A DIFFERENTIAL EQUATION WITH POWER-LAW DEGENERACY IN THE SPATIAL VARIABLE}

For $\alpha>0$, consider the equation

$$
u^{\prime \prime}(t)=t^{\alpha} A u(t), \quad t \geq 0 .
$$

If $A$ is the operator of differentiation with respect to the spatial variable $x$, for example, $A u(t, x)=u_{x x}^{\prime \prime}(t, x)$, then Eq. (30) is degenerating hyperbolically and generalizes the Tricomi equation but has another nature of degeneracy compared with the equations in the preceding sections. Therefore, it is natural to call the abstract equation (30) degenerating as well.

The change of the variable $t=(\tau / \mu)^{\mu}, \mu=2 /(\alpha+2)$, and of the unknown function $u(t)=(\tau / \mu)^{\mu} w(\tau)$ brings the degenerating equation (30) to the Euler-Poisson-Darboux equation

$$
w^{\prime \prime}(\tau)+\frac{\mu+1}{\tau} w^{\prime}(\tau)=A w(\tau), \quad \tau>0 .
$$

Since $0<\mu<1$, by Theorem 1 in [12] for $A \in G_{1-\mu} \subset G_{\mu+1}$ the function

$$
w(\tau)=\mu^{\mu} \tau^{-\mu} Y_{1-\mu}(\tau) u_{0}+Y_{\mu+1}(\tau) u_{1}
$$

is the unique solution of Eq. (31) with the two nonzero initial conditions

$$
\begin{aligned}
\lim _{\tau \rightarrow 0+}\left(w(\tau)-\mu^{\mu} \tau^{-\mu} Y_{1-\mu}(\tau) u_{0}\right) & =u_{1}, \\
\lim _{\tau \rightarrow 0+} \tau^{\mu+1} w^{\prime}(\tau) & =-\mu^{\mu+1} u_{0} .
\end{aligned}
$$

Getting back to the original variable in (32), (33), we obtain a representation of the solution of Eq. (30),

$$
u(t)=Y_{1-\mu}\left(\mu t^{1 / \mu}\right) u_{0}+t Y_{\mu+1}\left(\mu t^{1 / \mu}\right) u_{1},
$$

and the initial conditions

$$
u(0)=u_{0}, \quad u^{\prime}(0)=u_{1},
$$

that must be satisfied by this solution. We have thus established the following assertion.

Theorem 8. Let $\alpha>0, \mu=2 /(\alpha+2), A \in G_{1-\mu}$, and $u_{0}, u_{1} \in D(A)$. Then the function $u(t)$ defined by relation (34) is the unique solution of Eq. (30) with the initial conditions (35).

Note that in the special case of $A=A_{0}^{2}$ considered in [4], where $A_{0}$ is the generator of a $C_{0^{-}}$ group, there is no statement on uniqueness, and the proof of the assertion on solvability consists in verifying a representation of the form (3) for the Bessel operator function by means of differentiation in the integrand. Having the solution (34) and using the property of the Bessel operator function to determine a solution of the Euler-Poisson-Darboux equation, this verification can be carried out in a much simpler way.

We point out that adding "minor" terms to Eq. (30) requires, generally speaking, additional smoothness of the initial conditions compared with problem (30), (35), and the initial value problem for the modified equation may prove ill posed. Let us give an example. 
Example 3. Let $u_{0} \in D\left(A_{0}^{n}\right), n=\max \{2, m\}, m \in \mathbb{N}_{0}$, and let $A_{0}$ be the generator of the $C_{0}$-group $T\left(t ; A_{0}\right)$. Then the function

$$
u(t)=\sum_{j=0}^{m} \frac{m ! \sqrt{\pi} t^{2 j}}{j !(m-j) ! \Gamma(j+1 / 2)} T\left(t^{2} / 2\right) A_{0}^{j} u_{0}
$$

is a solution of the equation

$$
u^{\prime \prime}(t)=t^{2} A_{0}^{2} u(t)+(4 m+1) A_{0} u(t), \quad t \geq 0,
$$

with the initial conditions

$$
u(0)=u_{0}, \quad u^{\prime}(0)=0 .
$$

This fact is easy to check by substituting the function defined by relation (36) into Eq. (37) and then by matching the coefficients multiplying $t^{2 j} A_{0}^{j+1} u_{0}, 0 \leq j \leq m$, on the left- and right-hand sides in the resulting relation.

Relation (36) demonstrates the presence of a dependence between the coefficient multiplying $A_{0} u(t)$ in Eq. (37) and the smoothness of the initial element $u_{0}$ in the initial conditions (38) (cf. the case of a degenerating hyperbolic partial differential equation [3, p. 255]).

\section{DEGENERATE HYPERGEOMETRIC OPERATOR EQUATION}

In conclusion, let us show that, using fractional integro-differentiation (see [23, Sec. 2]), we can investigate the degenerating hypergeometric operator equation

$$
t u^{\prime \prime}(t)+(b I-t A) u^{\prime}(t)-c A u(t)=0, \quad t \geq 0,
$$

in which the parameters $b$ and $c$ satisfy the inequalities $b>c>0$.

We seek a solution, bounded at zero, of Eq. (39) and first assume that $b>1, c=\alpha$, and $0<\alpha<1$. Considering the relation (see [23, formula (15.11)])

$$
D_{0+}^{\alpha}(t u(t))=t D_{0+}^{\alpha} u(t)+\alpha D_{0+}^{\alpha-1} u(t)
$$

for the Riemann-Liouville fractional derivative $D_{0+}^{\alpha}$, we write this equation in the form

$$
D_{0+}^{\alpha}\left(t\left(D_{0+}^{1-\alpha} u(t)\right)^{\prime}+((b-\alpha) I-t A) D_{0+}^{1-\alpha} u(t)\right)=0 .
$$

Denoting $v(t)=D_{0+}^{1-\alpha} u(t)$, for the function $v(t)$ we obtain the equation

$$
t v^{\prime}(t)+(b-\alpha) v(t)=t A v(t)+t^{\alpha-1} v_{0}
$$

with some element $v_{0} \in E$.

For a solution of a first-order differential equation to exist, it is natural to assume that the operator $A$ is the generator of the $C_{0}$-semigroup $U(t ; A)$. Then for the solution of Eq. (40) we take the function

$$
v(t)=t^{\alpha-1} \int_{0}^{1}(1-s)^{b-2} U(t s ; A) v_{0} d s .
$$

Considering the representation (41), let us find a bounded solution of Eq. (39). After elementary 
transformations, we obtain

$$
\begin{aligned}
u(t) & =I_{0+}^{1-\alpha} v(t)=\frac{1}{\Gamma(1-\alpha)} \int_{0}^{t} \frac{v(\tau)}{(t-\tau)^{\alpha}} d \tau \\
& =\frac{1}{\Gamma(1-\alpha)} \int_{0}^{t} \frac{\tau^{\alpha-1}}{(t-\tau)^{\alpha}} \int_{0}^{1}(1-s)^{b-2} U(\tau s ; A) v_{0} d s d \tau \\
& =\frac{1}{\Gamma(1-\alpha)} \int_{0}^{t} \frac{\tau^{\alpha-b}}{(t-\tau)^{\alpha}} \int_{0}^{\tau}(\tau-x)^{b-2} U(x ; A) v_{0} d x d \tau \\
& =\frac{1}{\Gamma(1-\alpha)} \int_{0}^{t} U(x ; A) v_{0} \int_{x}^{t} \frac{\tau^{\alpha-b}(\tau-x)^{b-2}}{(t-\tau)^{\alpha}} d \tau d x \\
& =\frac{\Gamma(b-1)}{\Gamma(b-\alpha) t^{b-1}} \int_{0}^{t} x^{\alpha-1}(t-x)^{b-\alpha-1} U(x ; A) v_{0} d x \\
& =\frac{\Gamma(b-1)}{\Gamma(b-c)} \int_{0}^{1} s^{c-1}(1-s)^{b-c-1} U(t s ; A) v_{0} d s,
\end{aligned}
$$

where we have used integral 2.2.6.2 in [21].

Finally, for the resulting solution to satisfy the initial condition

$$
u(0)=u_{0} \in D(A),
$$

we set $v_{0}=\frac{\Gamma(b)}{\Gamma(b-1) \Gamma(c)} u_{0}$ in the representation (42). Then

$$
u(t)=\frac{\Gamma(b)}{\Gamma(c) \Gamma(b-c)} \int_{0}^{1} s^{c-1}(1-s)^{b-c-1} U(t s ; A) u_{0} d s .
$$

Relation (44), established for $b>1$ and $0<c<1$, also holds for $b>c>0$ by the analytic continuation principle. Thus, we have proved the following assertion.

Theorem 9. Let an operator $A$ generate a $C_{0}$-semigroup $U(t ; A), b>c>0$, and let the inclusion $u_{0} \in D(A)$ hold. Then the function $u(t)$ defined by relation (44) is a bounded solution of the degenerate hypergeometric operator equation (39) with the initial condition (43).

\section{FUNDING}

This work was supported by the Russian Foundation for Basic Research, project no. 19-01-00732.

\section{REFERENCES}

1. Smirnov, M.M., Vyrozhdayushchiesya ellipticheskie i giperbolicheskie uravneniya (Degenerating Elliptic and Hyperbolic Equations), Moscow: Nauka, 1966.

2. Smirnov, M.M., Uravneniya smeshannogo tipa (Mixed Type Equations), Moscow: Nauka, 1970.

3. Oleinik, O.A. and Radkevich, E.V., Uravneniya s neotritsatel'noi kharakteristicheskoi formoi (Equations with Nonnegative Characteristic Form), Moscow: Izd. Mosk. Univ., 2010.

4. Favini, A., Su un'equazione astratta di tipo ellittico-iperbolico, Rend. Sem. Mat. Univ. Padova, 1976, vol. 55, pp. 227-242. 
5. Vainerman, L.I., Hyperbolic equations with degeneracy in a Hilbert space, Sib. Mat. Zh., 1977, vol. 18, no. 4 , pp. $736-746$.

6. Orlov, V.P., Weakly degenerate hyperbolic equations, Differ. Equations, 2003, vol. 39, no. 10, pp. 1485-1496.

7. Goldstein, J.A., Semigroups of Linear Operators and Applications, Oxford-New York: Clarendon Press, 1985.

8. Glushak, A.V., The Bessel operator function, Dokl. Math., 1997, vol. 55, no. 1, pp. 103-105.

9. Glushak, A.V. and Pokruchin, O.A., Criterion for the solvability of the Cauchy problem for an abstract Euler-Poisson-Darboux equation, Differ. Equations, 2016, vol. 52, no. 1, pp. 39-57.

10. Brezis, H., Rosenkrantz, W., and Singer, B., On a degenerate elliptic-parabolic equation occurring in the theory of probability, Comm. Pure Appl. Math., 1971, vol. 24, pp. 395-416.

11. Glushak, A.V., Criterion for the solvability of the weighted Cauchy problem for an abstract EulerPoisson-Darboux equation, Differ. Equations, 2018, vol. 54, no. 5, pp. 622-632.

12. Glushak, A.V., Kononenko, V.I., and Shmulevich, S.D., A singular abstract Cauchy problem, Sov. Math., 1986, vol. 30, no. 6, pp. 78-81.

13. Glushak, A.V., Perturbations of an abstract Euler-Poisson-Darboux equation, Math. Notes, 1996, vol. 60, no. 3, pp. 269-273.

14. Glushak, A.V., The operator Bessel function, related semigroups, and the modified Hilbert transform, Differ. Equations, 1999, vol. 35, no. 1, pp. 130-132.

15. Levitan, B.M., Fourier series and integral expansions in Bessel functions, Usp. Mat. Nauk, 1951, vol. 1, no. 2(42), pp. 102-143.

16. Bragg, L.R., Fundamental solutions and properties of solutions of the initial value radial Euler-PoissonDarboux problem, J. Math. Mech., 1969, vol. 18, pp. 607-616.

17. Volk, V.Ya., On inversion formulas for a differential equation with a singularity at $x=0$, Usp. Mat. Nauk, 1953, vol. 8, no. 4(56), pp. 141-151.

18. Gel'fand, I.M. and Levitan, B.M., On the determination of a differential equation from its spectral function, Transl., Ser. 2, Am. Math. Soc., 1955, vol. 1, pp. 253-304.

19. Fomin, V.I., Vektornoe uravnenie Eilera vtorogo poryadka v banakhovom prostranstve (Vector SecondOrder Euler Equation in a Banach Space), Moscow: Spektr, 2012.

20. Watson, G.N., A Treatise on the Theory of Bessel Functions, Cambridge: Cambridge Univ. Press, 1944. Translated under the title: Teoriya besselevykh funktsii, Moscow: Izd. Inostr. Lit., 1949.

21. Prudnikov, A.P., Brychkov, Yu.A., and Marichev, O.I., Integraly i ryady. Elementarnye funktsii (Integrals and Series. Elementary Functions), Moscow: Nauka, 1981.

22. Dunaev, A.S. and Shlychkov, V.I., Spetsial'nye funktsii. Ch. 2 (Special Functions. Part 2), Yekaterinburg: Yurait, 2015.

23. Samko, S.G., Kilbas, A.A., and Marichev, O.I., Integraly i proizvodnye drobnogo poryadka $i$ nekotorye ikh prilozheniya (Fractional Integrals and Derivatives and Some of Their Applications), Minsk: Nauka Tekh., 1987. 